Check for updates

Cite this: RSC Adv., 2018, 8, 24002

\title{
Full-color tunable photoluminescent carbon dots based on oil/water interfacial synthesis and their applications $\uparrow$
}

\author{
Hongchong Guo, ${ }^{a}$ Bo You, (D) *a Shuyan Zhao, ${ }^{a}$ Yihao Wang, ${ }^{a}$ Gang Sun, ${ }^{b}$ Yujie Bai ${ }^{\mathrm{c}}$ \\ and Lei Shic
}

Herein, we developed a facile method to produce high yield, full-color tunable photoluminescent (PL) carbon dots (CDs) at the oil/water interface. This unique synthesis method for CDs involved the use of the oil-soluble small molecule styrene as both the reactant and oil phase medium at low temperature $\left(60{ }^{\circ} \mathrm{C}\right)$ through a catalytic-oxidation reaction in an aqueous system, which resulted in a high product yield (about $50 \mathrm{wt} \%$ ). Interestingly, the hydroxy-rich $\mathrm{CDs}$ at the oil/water interface self-assembled into nanospheres ( $\mathrm{N}$-CDs) spontaneously via hydrogen-bond interactions in the presence of styrene droplets. Without any surface passivation, the $\mathrm{N}-\mathrm{CD}$ s exhibited self-quenching-resistant and full-color tunable PL properties, which are highly desirable in the optoelectronic field. The mechanism of the formation of $\mathrm{N}$ CDs and their emission is proposed for the first time, which provides insight into the possible chemical processes occurring during the preparation. These distinctive CDs have potential novel applications in PL coatings, smart wearable optoelectronic devices, light emitting diodes, full-color displays, and solar cells.

Received 1st May 2018

Accepted 15th June 2018

DOI: $10.1039 / c 8 r a 03723 j$

rsc.li/rsc-advances
For example, most synthetic methods are unable to control the lateral dimensions and surface chemistry, and are unsuitable for the large-scale production of CDs. Furthermore, efficient and one-step strategies for the preparation of PL CDs are still lacking in this field. Currently, the fluorescence emission across the entire visible spectrum has been recognized as a key requirement for implementing CDs in nearly all practical applications. ${ }^{29-32}$ However, the as-prepared CDs covering the entire visible spectrum are still inadequate..$^{33-36}$ Moreover, nearly all CD-related research has focused on fluorescence from aqueous solution for bio-imaging or ion detection, while numerous applications, e.g., functional films, fabrics and optoelectronic devices and sensors, generally require PL materials emitting in the solid state. ${ }^{37-39}$ Thus, conventional CDs are unable to support these applications. Despite the great success in this area, it is still urgent to develop novel techniques for the synthesis of high yield, full-color tunable PL CDs with unique properties for photoelectric applications.

Oil/water interfacial synthesis is usually used for the fabrication of nanocrystals such as noble metal ( $\mathrm{Au}, \mathrm{Pt}$, and $\mathrm{Ag}$ ), semiconductors (CdS, $\mathrm{PbS}$, and $\mathrm{ZnS}$ ), rare earth fluorescence $\left(\mathrm{TiO}_{2}, \mathrm{CuO}, \mathrm{ZrO}_{2}, \mathrm{SnO}_{2}\right.$ and $\left.\mathrm{ZnO}\right)$, and magnetic nanocrystals $\left(\mathrm{CoFe}_{2} \mathrm{O}_{4}\right) \cdot{ }^{40}$ Meanwhile, the lower surface tension and minimized Helmholtz free energy of interfacial systems cause nanoparticles to self-assemble into thin films (nano-layers), vesicles and other two-dimensional (2D) structures for a broad range of applications such as electronics, optics, catalysis and sensors. ${ }^{\mathbf{4 1 3}}$ The interfacial synthesis and self-assembly of nanomaterials is facile, green, easy controllable and operation. 
However, this method has rarely been reported for the fabrication of CDs.

Herein, we present a facile and one-step strategy for the synthesis of PL CDs at the oil/water interface using the oilsoluble small molecule styrene as both the reactant and oil phase medium, which results in a high product yield ( $c a$. 50 wt $\%)$ and distinctive characteristics. Different from the conventional interfacial synthesis, this strategy uses the small organic molecule styrene as both the reactant and oil phase medium, copper sulfate $\left(\mathrm{CuSO}_{4}\right)$ as the catalyst dissolved in the water phase and hydrogen peroxide $\left(\mathrm{H}_{2} \mathrm{O}_{2}\right)$ as the oxidant and the initiator. The products are generated at the interface of the styrene droplets and water through a catalytic-oxidation process at a reaction temperature as low as $60{ }^{\circ} \mathrm{C}$. Besides, other benzene series including benzene, benzyl alcohol, xylene can also be converted to CDs under the same conditions. Since the final products are CDs and water, this reaction is environmentally friendly and significant. Interestingly, the high concentration of CDs at the oil/water interface self-assemble into nanospheres (N-CDs). The PL emission of the N-CDs can be tuned by varying the reaction time, temperature and concentration of oxidants, resulting in self-quenching-resistant and full-color tunable PL properties (400 to $800 \mathrm{~nm}$ ). Without any surface passivation, the acquired products exhibit good dispersion in waterborne polymer latex systems and polar organic solvents (such as methanol, ethanol, acetonitrile, acetone tetrahydrofuran, and methylene chloride). Moreover, the colloidal CD ethanol solution can also be used to dye various textiles knitted from PET, nylon, cotton and iron wire without fluorescent quenching, which is extremely promising for many applications, e.g., light-emitting diodes, full-color displays, and optoelectronic devices.

\section{Experimental}

In a typical procedure for the synthesis of diamond-like CDs, styrene $(0.208 \mathrm{~g}$, Aladdin, purity $>98 \%)$ and $\mathrm{H}_{2} \mathrm{O}_{2}(400 \mathrm{mM})$ were added to a $\mathrm{CuSO}_{4}(20 \mathrm{mM})$ solution of DI water $(10 \mathrm{~mL})$ at $60{ }^{\circ} \mathrm{C}$ under stirring for $12 \mathrm{~h}$. The final aqueous dispersion of brown particles was purified by centrifugation and washed with water several times to remove the supernatant including the residual organic molecules and inorganic impurities. Then the purified brown CDs were dried at $60{ }^{\circ} \mathrm{C}$ for structural characterization and measurements. The synthetic procedure for the CDs using other benzene compounds was similar to that of the styreneCDs except for the addition of different benzene compounds in the same media: $0.2 \mathrm{~mol} \mathrm{~L}^{-1}$ benzene, $0.2 \mathrm{~mol} \mathrm{~L}^{-1}$ benzyl alcohol and $0.2 \mathrm{~mol} \mathrm{~L}^{-1}$ xylene.

TEM and HRTEM observations were performed on an aberration-corrected TEM (FEI Tecnai G2 F20 S-Twin) operating at an acceleration voltage of $200 \mathrm{kV}$. Absorption and fluorescence spectra were recorded at room temperature on a Hitachi 4100 spectrophotometer and QM40 fluorescence spectrophotometer, respectively. ${ }^{13} \mathrm{CNMR}$ spectra of the sample powders were collected on a 400M Bruker AVANCE III HD instrument. ${ }^{13} \mathrm{C}$-NMR spectra of the CD powder and liquid ${ }^{1} \mathrm{H}-\mathrm{NMR}$ spectroscopic measurement were collected on a 400M Bruker
AVANCE III HD instrument. FT-IR spectra of dried sample powders were recorded with a Bruker VERTEX 70 spectrometer. Raman spectra were recorded on a XploRA in plus laser Raman spectrometer with lexc 1/4 $638 \mathrm{~nm}$. XPS spectra were collected using a PHI 5000C \& PHI5300 X-ray photoelectron spectrometer. The PL QY of CD ethanol solutions from the benzene series was determined by comparing the integrated PL intensities (excited at $365 \mathrm{~nm}$ ) and the absorbance values (at $365 \mathrm{~nm}$ ) using quinine sulfate in $0.10 \mathrm{mmol} \mathrm{L}^{-1} \mathrm{H}_{2} \mathrm{SO}_{4}$ as the reference.

\section{Results and discussion}

The preparation of the CDs was readily accomplished, where the oil-soluble small molecule styrene was added to deionized water first, which formed a two-phase system including an oil phase and a transparent aqueous phase. Then, $\mathrm{CuSO}_{4}$ and $\mathrm{H}_{2} \mathrm{O}_{2}$ were used to initiate the reaction at $60{ }^{\circ} \mathrm{C}$ (Fig. 1a). The mixture solution was stirred for the entire process. After 30 minutes, the solution turned yellow and egg-yellow products were suspended on the surface of the solution. About six hours later, more and more brown product was suspended on the oil/water interface, as shown in Fig. 1a. The products were purified by centrifugation and washed with water several times to remove the supernatant including the residual organic molecules and inorganic impurities, and then dried in a vacuum oven.

The morphology of the CDs was characterized via transmission electron microscopy (TEM) and high resolution transmission electron microscopy (HRTEM), as shown in Fig. 1b-d. The HRTEM images indicate that the size of a single carbon dot is about $2-3 \mathrm{~nm}$ and several carbon dots "hand in hand" selfassembled into nanospheres (N-CDs) with diameters in the range of about 50-200 $\mathrm{nm}$ (Fig. 1c). The obvious lattice spacing is observed to be about $0.208 \mathrm{~nm}$, and the selected area electron diffraction (SAED) pattern reveals that the ratio of squares of the ring radius is $3: 8: 11$, which corresponds to the $\{111\},\{220\}$, and $\{311\}$ planes of cubic diamond (the inset of Fig. 1d). These results indicate the as-synthesized CDs might have a diamondlike crystalline structure. ${ }^{\mathbf{4 4 - 4 6}}$ The ultraviolet-visible (UV-Vis) absorption spectrum of the CDs display a broad absorption band (200 to $800 \mathrm{~nm}$ ) (Fig. 1e), which indicates a broad size distribution in this system, which is a general phenomenon of the bottom-up synthetic strategy. ${ }^{47}$ Additionally, the present method can also be applied to other oil-soluble small molecules in the benzene family such as benzene, benzyl alcohol and xylene (see the ESI, Fig. S1†). Without any surface passivation, the PL quantum yields of the CDs synthesized using benzene, styrene, benzyl alcohol and xylene were 0.26, 2.44, 0.39 and 0.43, respectively, under $360 \mathrm{~nm}$ excitation using quinine sulfate in $0.10 \mathrm{mmol} \mathrm{L}^{-1} \mathrm{H}_{2} \mathrm{SO}_{4}$ as a reference (Fig. $\mathrm{S} 2 \dagger$ ). ${ }^{48}$ These values are much higher than that of CDs without surface passivation. ${ }^{47,49}$ Photographs of the terminal reaction solution and the powders of the CDs synthesized using different benzene compounds as the carbon source are shown in Fig. 1f and g, respectively.

Furthermore, the PL emission spectra obviously show that CDs have multi-emission properties, which depend on the excitation wavelength (Fig. 2a). Upon excitation of the CDs at 
a

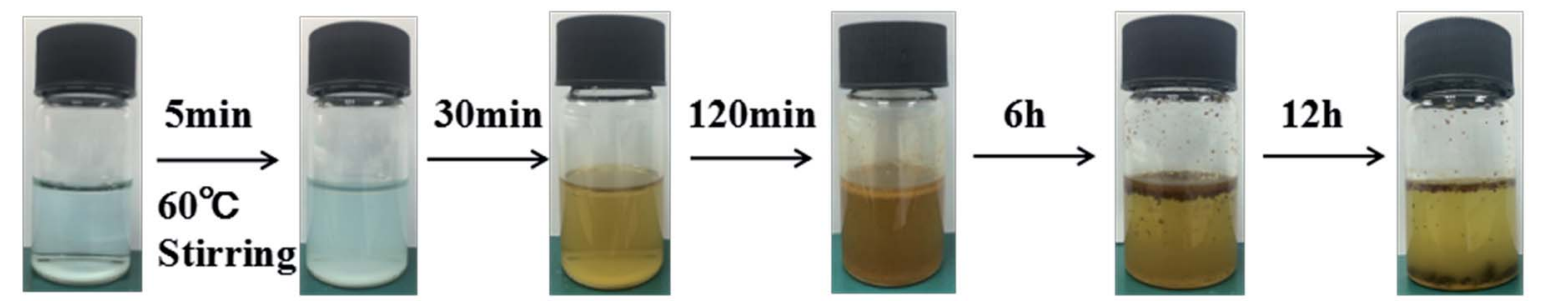

b
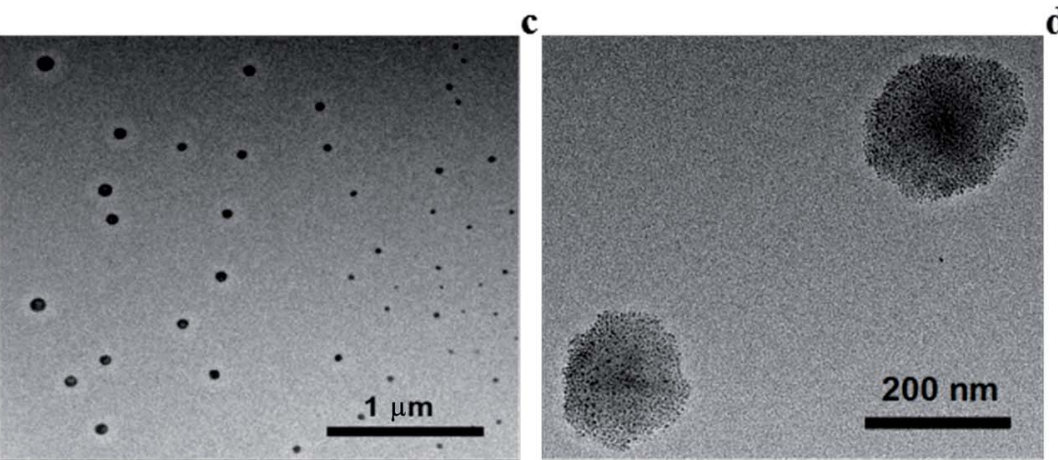

d

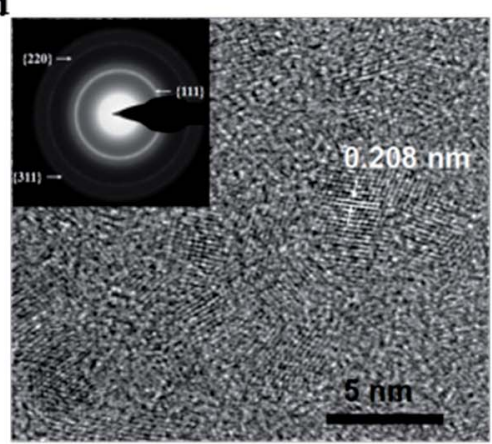

e

f
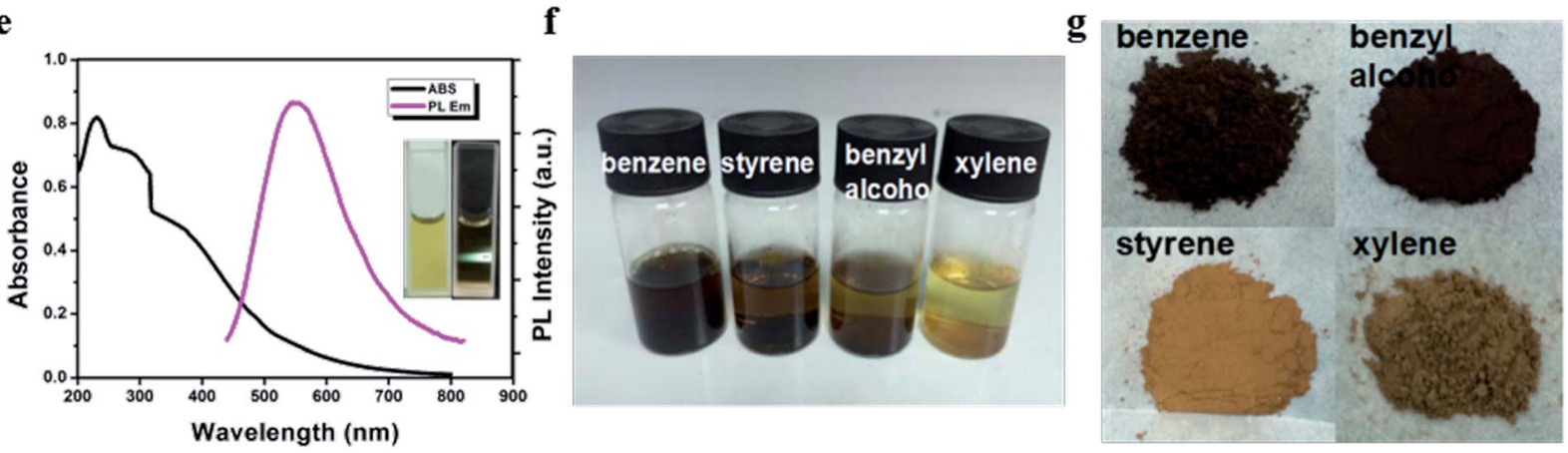

Fig. 1 Preparation and structural characterization of CDs. (a) synthetic procedure; (b) TEM images of CDs; (c) HRTEM images and SAED of CDs; (d) UV/Vis absorption spectrum and PL emission spectra of a raw CDs aqueous solution excited at 420 nm (inset: photographs of a raw CDs aqueous solution under sunlight and $405 \mathrm{~nm}$ laser pointer). (e) Optical photographs of the terminal reaction solution (after $24 \mathrm{~h}$ ) (f) and (g) the powders of the carbon dots synthesized by benzene, styrene, benzyl alcohol and xylene.

varying wavelengths ranging from 300 to $600 \mathrm{~nm}$, their emitted fluorescence maximum red shifted from 400 to $800 \mathrm{~nm}$ and the highest fluorescence intensity was observed at $420 \mathrm{~nm}$ excitation. The Raman spectrum shows a D band at $1360 \mathrm{~cm}^{-1}$, which reflects the disorder or defects in the graphite carbon or glassy carbon, and a strong signal for the $\mathrm{G}$ band assigned to the $\mathrm{sp}^{2}$ carbons at $1590 \mathrm{~cm}^{-1}$ (Fig. 2b). ${ }^{50}$ This suggests that the CDs may contain a percentage of graphite structure on the surfaces of diamond cores.51,52 Moreover, the existence of $\mathrm{sp}^{3}$-and $\mathrm{sp}^{2}$ hybridized carbon atoms is confirmed from the solid ${ }^{13} \mathrm{C}$ NMR spectrum (Fig. 2c). The signals in the range of $25-50 \mathrm{ppm}(\delta=$ $38 \mathrm{ppm})$ correspond to aliphatic $\left(\mathrm{sp}^{3}\right)$ carbon belonging to the diamond core, ${ }^{53-55}$ while that in the range of 50-100 $\operatorname{ppm}(\delta=79$ ppm) are assigned to oxygen-doped carbon atoms modified on the surface of the diamond cores. The signals in the range of 90-150 $\mathrm{ppm}$ indicate $\mathrm{sp}^{2}$-hybridized carbon atoms. Besides the ${ }^{13} \mathrm{C}$ NMR spectrum, the ${ }^{1} \mathrm{H}$ NMR spectrum further verifies the $\mathrm{sp}^{2}$ aromatic proton resonance signals of the CDs, which are in the range of 6-8 ppm. Also, the signals in the range of $150-$ $200 \mathrm{ppm}$ in the ${ }^{13} \mathrm{C}$ NMR spectrum are mainly attributed to the carbonyl groups on the surface of the CDs (Fig. S3 $\uparrow$ ). Based on these results, the CDs obtained most probably consist of a nanocrystalline $\mathrm{sp}^{3}$ core that features diamond carbon and a graphitic shell, which are stabilized by transforming into $\mathrm{sp}^{2}$ carbons and functionalization with carbonyl moieties on the particle surface.

The FTIR spectrum (Fig. 2d) also shows that the surface of the CDs has abundant oxygen-containing functional groups. The broad absorption band at $3100-3500 \mathrm{~cm}^{-1}$ is assigned to $\nu(\mathrm{O}-\mathrm{H})$, and the absorption peaks at $1717 \mathrm{~cm}^{-1}$ and $1234 \mathrm{~cm}^{-1}$ are attributed to the $\mathrm{C}=\mathrm{O}$ and $\mathrm{C}-\mathrm{O}-\mathrm{C}$ groups, respectively, which indicate the CDs have an oxidized surface. Furthermore, the peaks at $1616 \mathrm{~cm}^{-1}$ and $835 \mathrm{~cm}^{-1}$ are assigned to the $\mathrm{sp}^{2}$ aromatic $\mathrm{C}=\mathrm{C}$ units and aromatic $\mathrm{CH}_{2}$ rocking, respectively. The asymmetric and symmetric stretching, and out-of-plane and in-plane bending vibrations of $\mathrm{CH}_{3}$ and $\mathrm{CH}_{2}$ on the $\mathrm{CDs}$ are located at 2926, 2855, 1375 and $1454 \mathrm{~cm}^{-1}$ bands. The bands in the range of $1000-1300 \mathrm{~cm}^{-1}$ correspond to the $\mathrm{C}-\mathrm{OH}$ stretching and $-\mathrm{OH}$ bending vibrations, which imply the existence of a large number of residual hydroxyl groups. ${ }^{56}$ The typical X-ray photoelectron spectroscopy (XPS) data shows that the CDs only contain two elements, about $77 \%$ carbon and $23 \%$ 
a

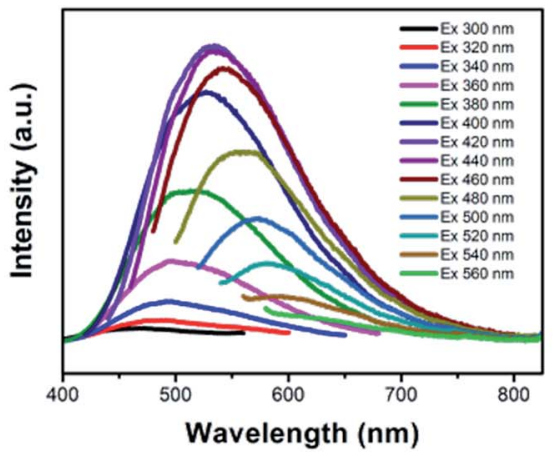

c

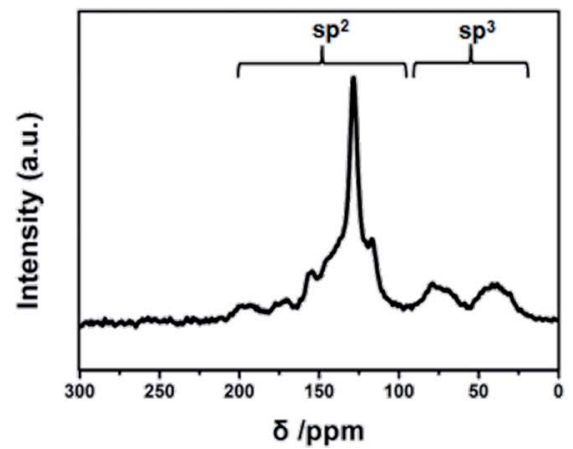

e

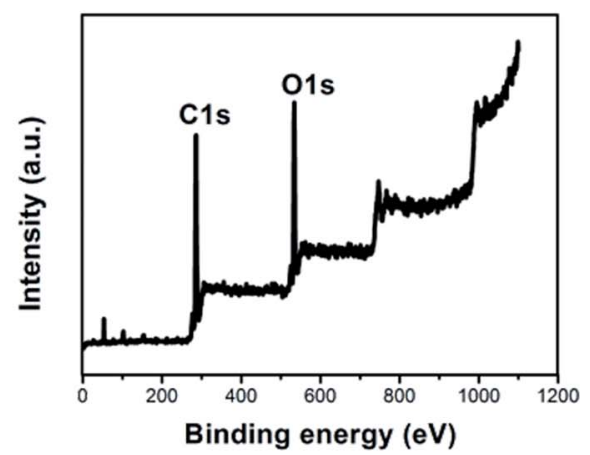

b

d
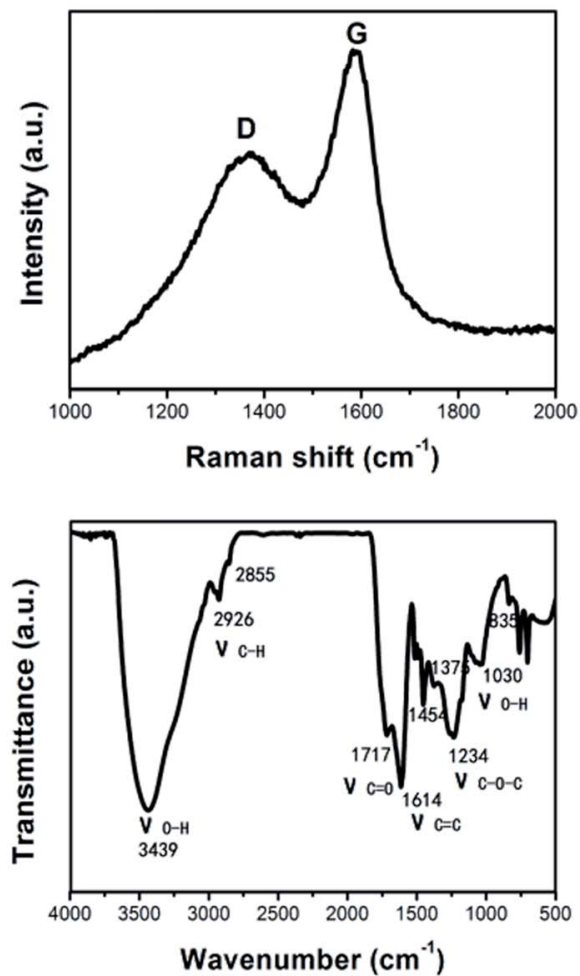

f

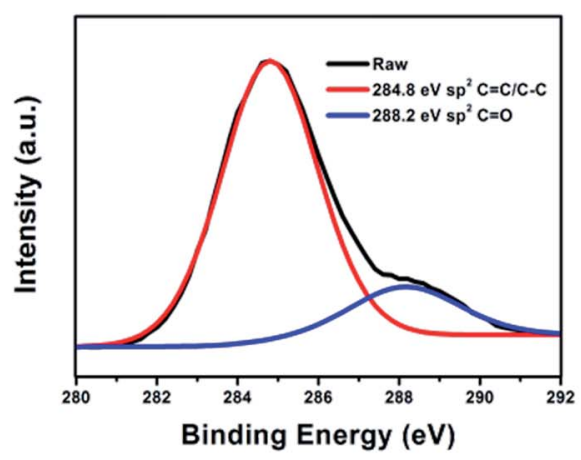

Fig. 2 Characterization of the microstructure and surface state of the as-synthesized CDs. (a) PL emission under different excitation wavelengths, (b) Raman spectrum, (c) ${ }^{13} \mathrm{C}-\mathrm{NMR}$ spectrum, (d) FTIR spectrum, (e) XPS spectrum and (f) C 1s XPS spectrum of the CDs. The sample was prepared using styrene with $400 \mathrm{mM} \mathrm{H}_{2} \mathrm{O}_{2}$ and $20 \mathrm{mM} \mathrm{CuSO}_{4}$.

oxygen (Fig. 2e). The $\mathrm{C}$ 1s spectrum reveals two carbon atom peaks at ca. $284.8 \mathrm{eV}(\mathrm{C}=\mathrm{C}$ or $\mathrm{C}-\mathrm{C})$ and $288.2 \mathrm{eV}(\mathrm{C}=\mathrm{O})$ (Fig. 2f). All these results prove the chemical structure and surface functional groups involved in the CDs, which are favorable for their stability in aqueous systems.

Furthermore, the intrinsic PL properties of the CDs were thoroughly investigated. The PL decay trace of the CDs in ethanol solution could be fitted well using a mono-exponential function with an average lifetime in the range of 2.22-2.43 ns, which were prepared with $\mathrm{s}$ lower degree of oxidation ( $<600 \mathrm{mmol} \mathrm{L}^{-1} \mathrm{H}_{2} \mathrm{O}_{2}$ ) (Table $\mathrm{S} 1 \dagger$ ). In contrast, the PL decay of the CDs with a higher degree of oxidation $\left(>600 \mathrm{mmol} \mathrm{L}^{-1}\right.$ $\mathrm{H}_{2} \mathrm{O}_{2}$ ) is fitted well to a bi-exponential function with an increased average lifetime of $2.50 \mathrm{~ns}$, which is comparable to that of previously reported CDs (Fig. S4a $\dagger$ ). ${ }^{57}$ This further demonstrates that the emission is contributed by two components, i.e., surface fluorophores and diamond cores. Besides, they demonstrated high photostability for a period of $1000 \mathrm{~s}$ (Fig. S4b $\dagger$ ) and possessed reversible thermo-sensitivity when the temperature varied from $293 \mathrm{~K}$ to $393 \mathrm{~K}$ (Fig. S4c $\dagger$ ). Another interesting phenomenon of this $\mathrm{pH}$-dependent behavior is that the PL intensities of the CDs decrease in solution with a high or low $\mathrm{pH}$ value, whereas they remain almost stable when the $\mathrm{pH}$ changes from 5 to 9 (Fig. $\mathrm{S} 4 \mathrm{~d} \dagger$ ).

To gain more insight into the origin of the CDs, we tried different techniques to illustrate the possible mechanism of their formation. HRTEM was employed to observe the growth of the CDs. At the beginning of the reaction with $\mathrm{H}_{2} \mathrm{O}_{2}$ oxidation, a few CDs were generated. Subsequently, with an increase in reaction time, large quantities of CDs were produced, and self- 
assembled into nanospheres (N-CDs) simultaneously (Fig. 3ac). Additionally, their time-dependent UV-Vis absorption spectra were also monitored during the initial process, which indicate an increase in UV-Vis absorption $(<600 \mathrm{~nm})$ with an increase in the amount of nanoparticles produced (Fig. S5†). Meanwhile, the fluorescence emission intensity was enhanced until the maximum at 210 minutes and then declined slightly (Fig. 3d). This suggests that the high concentration of CDs contributed to the increased luminescence intensity until the intensity almost did not change, which implies high photostability without self-quenching. Fig. $3 \mathrm{~g}$ shows the possible dynamic process. Since the oil-soluble styrene is not miscible with water by mixing, two phases were formed initially. After continuous stirring, styrene formed a variety of oil-droplets with different sizes in the water phase as both the reactant and oil phase medium. With the addition of $\mathrm{CuSO}_{4}$ and $\mathrm{H}_{2} \mathrm{O}_{2}$, CDs were produced at the interface of the styrene droplets and water via a catalytic-oxidation process. Under $\mathrm{H}_{2} \mathrm{O}_{2}$ oxidation, more and more CDs were endowed with large quantities of carbonyl and hydroxy groups. Finally, the hydroxy-rich CDs at the oil/water interface self-assembled into nanospheres via hydrogen-bond interactions until the styrene droplets were totally converted into $\mathrm{N}$-CDs in reaction equilibrium, while maintaining the minimum Helmholtz free energy. ${ }^{58}$

To the best our knowledge, the reaction mechanism of the as-produced CDs formed in the $\mathrm{CuSO}_{4} / \mathrm{H}_{2} \mathrm{O}_{2}$ catalytic-oxidation system is notably different from the mechanism that usually explains the formation of iron-based photo-Fenton systems. ${ }^{59}$ The possible nucleation mechanism of the CDs may be that under $\mathrm{CuSO}_{4}$ catalysis, $\mathrm{H}_{2} \mathrm{O}_{2}$ is rapidly decomposed, which produces a large amount of active intermediate $\cdot \mathrm{OH}$ species in water when the $\mathrm{pH}$ is about $3-4$ (Fig. $\mathrm{S} 6 \dagger$ ) and then the solution turns yellow. ${ }^{60}$ The possible reaction proposed is as follows ((1)$(4)):^{61}$

$$
\begin{gathered}
\mathrm{Cu}(\mathrm{II})+\mathrm{H}_{2} \mathrm{O}_{2} \rightleftharpoons \mathrm{Cu}(\mathrm{I})+\mathrm{HO}_{2}{ }^{\circ}+\mathrm{H}^{+} \\
\mathrm{HO}_{2}{ }^{\cdot} \rightleftharpoons \mathrm{O}_{2}^{\cdot-}+\mathrm{H}^{+}\left(\mathrm{p} K_{\mathrm{a}}=4.8\right) \\
\mathrm{Cu}(\mathrm{II})+\mathrm{O}_{2}^{\cdot-}\left(\text { or } \mathrm{HO}_{2}{ }^{\cdot}\right) \rightleftharpoons \mathrm{Cu}(\mathrm{I})+\mathrm{O}_{2}\left(\text { or } \mathrm{O}_{2}+\mathrm{H}^{+}\right) \\
\mathrm{Cu}(\mathrm{I})+\mathrm{H}_{2} \mathrm{O}_{2} \rightarrow \mathrm{Cu}(\mathrm{II})+{ }^{\cdot} \mathrm{OH}+\mathrm{OH}^{-}
\end{gathered}
$$

In the first step, $\mathrm{Cu}$ (II) is reduced to $\mathrm{Cu}(\mathrm{I})$ by $\mathrm{H}_{2} \mathrm{O}_{2}$, which produces a hydroperoxyl radical $\left(\mathrm{HO}_{2}{ }^{\circ}\right)$ (reaction (1)). $\mathrm{HO}_{2}{ }^{\circ}$ or its a

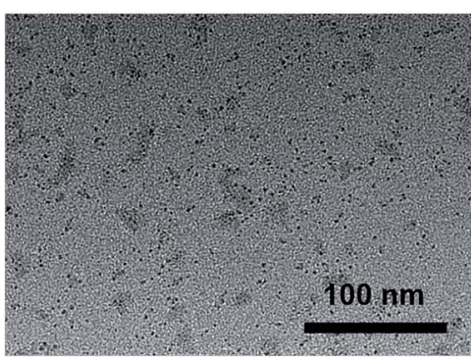

d

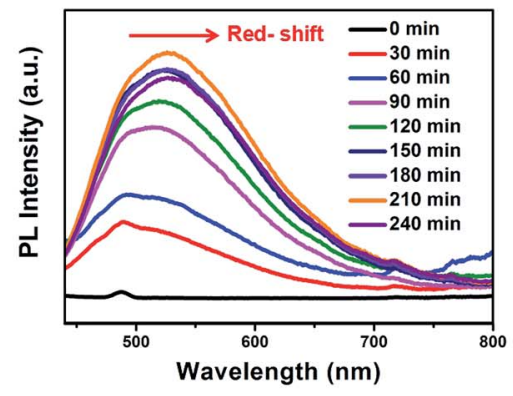

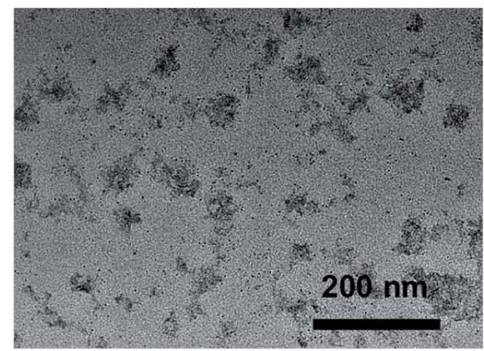

e

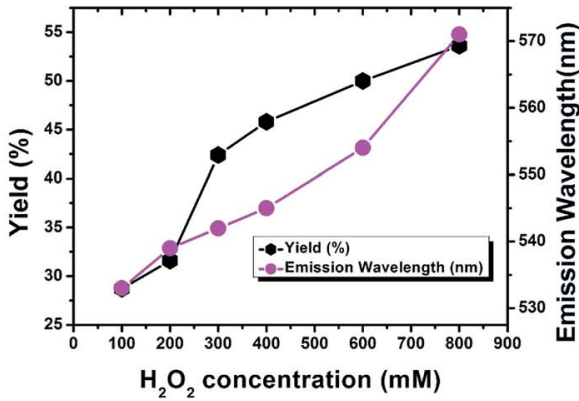

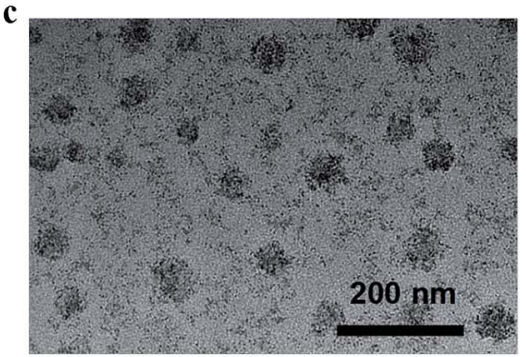

f

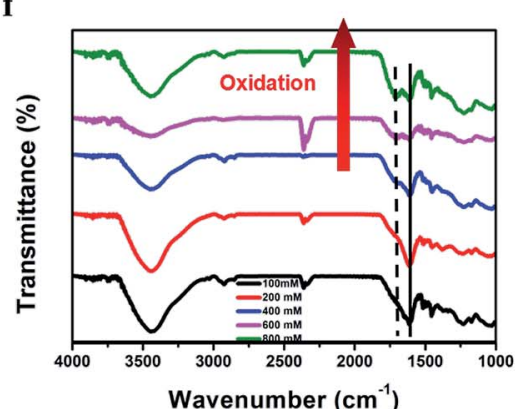

g
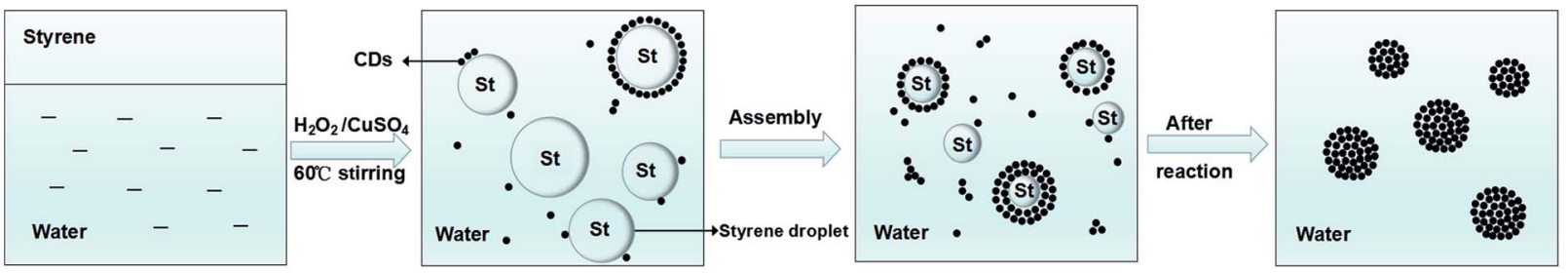

Fig. 3 HRTEM image of the CDs formed at (a) $30 \mathrm{~min}$ (b) $120 \mathrm{~min}$ and (c) $180 \mathrm{~min}$. (d) PL emission spectra of the CDs as a function of reaction time. (e) Yield and PL emission wavelength versus concentration of $\mathrm{H}_{2} \mathrm{O}_{2}$ excited at $420 \mathrm{~nm}$ in ethanol solution. (f) FTIR spectra with different concentrations of $\mathrm{H}_{2} \mathrm{O}_{2}\left(100 \mathrm{mmol} \mathrm{L}^{-1}, 200 \mathrm{mmol} \mathrm{L}^{-1}, 400 \mathrm{mmol} \mathrm{L}^{-1}, 600 \mathrm{mmol} \mathrm{L}^{-1}\right.$, and $\left.800 \mathrm{mmol} \mathrm{L}^{-1}\right)$. (g) Schematic for the formation of NCDs. 
conjugate base (superoxide radical anion, $\mathrm{O}_{2}{ }^{-}$) can reduce another molecule of $\mathrm{Cu}$ (II) (reaction (3)). Then, the irreversible reaction of $\mathrm{Cu}(\mathrm{I})$ with $\mathrm{H}_{2} \mathrm{O}_{2}$ produces $\cdot \mathrm{OH}$. In this process, the overall kinetics depends on the rate of the reduction of $\mathrm{Cu}$ (II) to $\mathrm{Cu}(\mathrm{I})$, which is governed by reaction (1) (i.e., the ratedetermining step). Upon mechanical agitation and active group oxidation, many styrene droplets are decomposed to form $\mathrm{C}_{2}, \mathrm{C}_{3}, \mathrm{C}_{2} \mathrm{H}_{2}$, and other small radicals (Fig. $3 \mathrm{~g}$ ). ${ }^{62}$ With the significant production of $\mathrm{H}^{+}$, the acidity of the solution increases $(\mathrm{pH}<2)$ (Fig. $\mathrm{S} 6 \dagger)$ and some small radicals rapidly form dense carbon clusters and crystallize into CDs rather than be further oxidized into $\mathrm{CO}_{x}$. This is due to the slow generation rate of $\cdot \mathrm{OH}$ in acidic media upon the reversible reaction process of the intermediate $\mathrm{Cu}$ (II) with $\mathrm{H}_{2} \mathrm{O}_{2}$. Some water soluble organics (such as citric acid, ascorbic acid, and ethylene glycol) can also be carbonized to CDs by this catalytic-oxidation method (see Fig. S7†). Therefore, surface-bonded hydrogen, which remains from the hydrocarbon molecules, contributes to the irregular shape of the CD particles, as previously reported. Furthermore, due to the presence of surface hydrogen, the CDs are energetically favoured over aromatic compounds without the need for high pressures or extreme kinetic conditions. ${ }^{\mathbf{6 3 , 6 4}}$ After continuous oxidization, the $\mathrm{sp}^{3}$ diamond cores finally stabilize through termination with some percentage of graphite shell $\mathrm{sp}^{2}$ carbon. ${ }^{65}$ Thus, the formation of graphite (amorphous carbon) and diamond is possible, and the yield of which may be determined by reaction kinetics. ${ }^{66}$ Furthermore, the fluorescence wavelength of the CDs obtained at a given time displayed a red-shift as larger N-CDs were considered (Fig. 3d). These results illustrate that the $\mathrm{N}-\mathrm{CDs}$ in this system may play a key role in the fluorescence emission, and the size of the N-CDs might affect the red-shift of the fluorescence wavelength maxima. Accordingly, we speculate that the reason why and how the CDs can resist self-quenching is probably as follows: firstly, the diamond cores with hardness cannot directly contact with each other, and the repulsion keeps the CDs an appropriate interval (within the Förster distance, $R_{0}$ ) between the inner particles ${ }^{67}$ Secondly, the self-assembled CDs as building blocks interacted through weak forces: van der Waals and hydrogen bonding. The formed N-CDs enhanced luminescence intensity more than the single carbon dots and induced a red-shift om the emission. Some researchers have attributed this phenomenon to the conjugate effect or quantum-size effect; ${ }^{68,69}$ nevertheless, the mechanism remains an open question. Therefore, compared with the conventional graphitized CDs, the existence of diamond-like cores prevented $\pi-\pi$ interactions in the pure graphitic structure, and the N-CDs resisted self-quenching in the aggregation state in the aqueous system and give rise to a red-shift in the emission simultaneously. In other words, without any complex surface modification to solve the issue of CD aggregation, self-quenching can be effectively avoided, which reduces the cost compared with existing methods.

Our results also show the surface-state-tunable PL emission of CDs with different degrees of surface oxidation, which is consistent with previous reports. ${ }^{70}$ Fig. 3e displays not only that a high yield of CDs can be acquired by increasing the concentration of $\mathrm{H}_{2} \mathrm{O}_{2}$, but also the fluorescence peak wavelength maximum red-shifts. However, no obvious changes are observed when more $\mathrm{CuSO}_{4}$ is added under the same conditions (Fig. S8a and S8b $\dagger$ ). The FTIR spectra prove that the carbonyl group $(\mathrm{C}=\mathrm{O})$ was gradually formed on the surface of the CDs by oxidation with a higher concentration of $\mathrm{H}_{2} \mathrm{O}_{2}$ (Fig. 3f). ${ }^{71}$ This means that the predominant emission with the maximum transition on the CDs is probably associated with $\mathrm{H}_{2} \mathrm{O}_{2}$ oxidation through the one-step oxidation process. The higher concentration of $\mathrm{H}_{2} \mathrm{O}_{2}$ may result in a higher degree of surface oxidation states, which further facilitate the formation of CDs with long-wavelength emissions. Additionally, the intensity ratios of the D-bands to G-bands $\left(I_{\mathrm{D}}\right.$ to $\left.I_{\mathrm{G}}\right)$ of the Raman signal of the CDs oxidized by increasing the concentration of $\mathrm{H}_{2} \mathrm{O}_{2}$ were $0.42,0.47,0.49,0.54$, and 0.56 , respectively (Fig. S9†), which indicates a growing fraction of defects on the CDs. ${ }^{70}$ The surface defects created by surface oxidation serve as capturing centers of excitons, thereby giving rise to surface-state-related fluorescence. Therefore, the higher degree of surface oxidation, the more surface defects on the CDs. Consequently, this change narrows the energy levels and causes a red-shifted emission in the CDs. ${ }^{72,73}$ All these results manifest that the emission is possibly attributed to the surface oxidation state of the CDs, and a higher degree of the surface oxidation resulted in a longer wavelength emission. ${ }^{74}$ Therefore, controllable full-color PL CDs can be acquired by tuning the size and surface oxidation degree of the N-CDs. ${ }^{75,76}$

Interestingly, we noticed that the optimal emission was redshifted from $450 \mathrm{~nm}$ and $560 \mathrm{~nm}$ to $660 \mathrm{~nm}$, when the CD powder was re-dispersed in ethanol with an increased solute concentration (Fig. S10a-d†), which means full-color PL emission can be realized by varying the CD concentration, which is more controllable than the use of excitation-independent crystalline organic molecules. ${ }^{77,78}$ Besides, distinctive luminescence emission of the CDs in different polar solvents (such as methanol, ethanol, acetonitrile, acetone tetrahydrofuran, and methylene chloride) could be achieved (Fig. S10f $\dagger$ ), which also confirms their high content of surface polar organic groups. ${ }^{79}$ Therefore, CDs/polyacrylate coating films can be fabricated by mixing the CDs with polyacrylate latex in different ratios. The coating films are so transparent with a good optical properties that the Fudan Logo is clearly observed on a metallic substrate in the visible light region, with a transmittance $(T)$ of $c a .80 \%$ at in the wavelength range of $400-800 \mathrm{~nm}$ for a $1.33 \mathrm{wt} \%$ concentration of CDs in the coating film, which is much higher transparency than that of other CD composites (Fig. 4a-f and Fig. S11a $\dagger) .{ }^{80}$ Hence, the good transparency of the coating films further demonstrates the uniform dispersion of CDs in the waterborne polyacrylate latex and complete absence of agglomerated CDs in the polymer matrix, which avoids the usual Rayleigh scattering and luminescence quenching. ${ }^{81}$

Significantly, bright visible blue, green, yellow and red emission from the CDs/polyacrylate fluorescence coating films were achieved upon excitation by a single wavelength (Fig. 4i-v), while the pure polyacrylate latex coating films could only reflect ultraviolet light (Fig. 4ii). As the loading fraction of CDs increased, color changes were observed (Fig. 4iii-iv). Additionally, the fluorescent coatings can be painted on the substrate, 


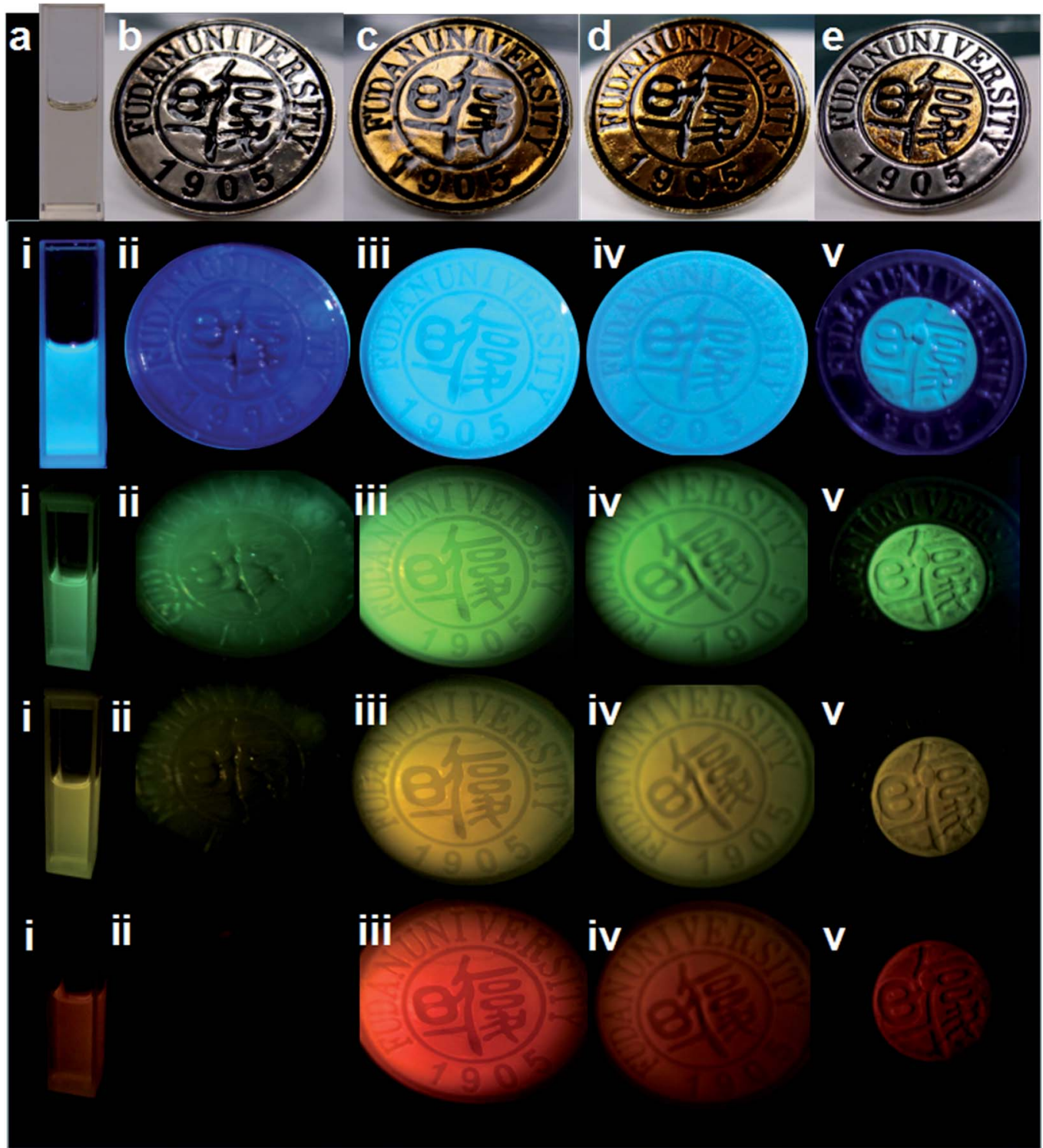

f

Fig. 4 Optical photographs of acrylic resin doped with various ratios of carbon dots upon visible light (a-f) and full-color fluorescent coatings (blue, green, yellow, red CDs with UV light (i-v): (a) CDs in ethanol. (b) Acrylic resin without CDs (control). (c) Acrylic resin with 0.67 wt\% CDs. (d) $1.33 \%$ CDs. (e) $1.33 \%$ CDs acrylic resin coated on the middle of the metallic school badge $(d=2 \mathrm{~cm})$. (f) large-area coatings ( $1.33 \% \mathrm{CDs}) 30 \mathrm{~mL}$ on the $50 \times 32 \mathrm{~cm}$ PET films under visible light. The fluorescence microscopy images (green, yellow, red) were obtained through band-pass filters at different wavelengths: $500 \mathrm{~nm}, 550 \mathrm{~nm}$, and $600 \mathrm{~nm}$. 

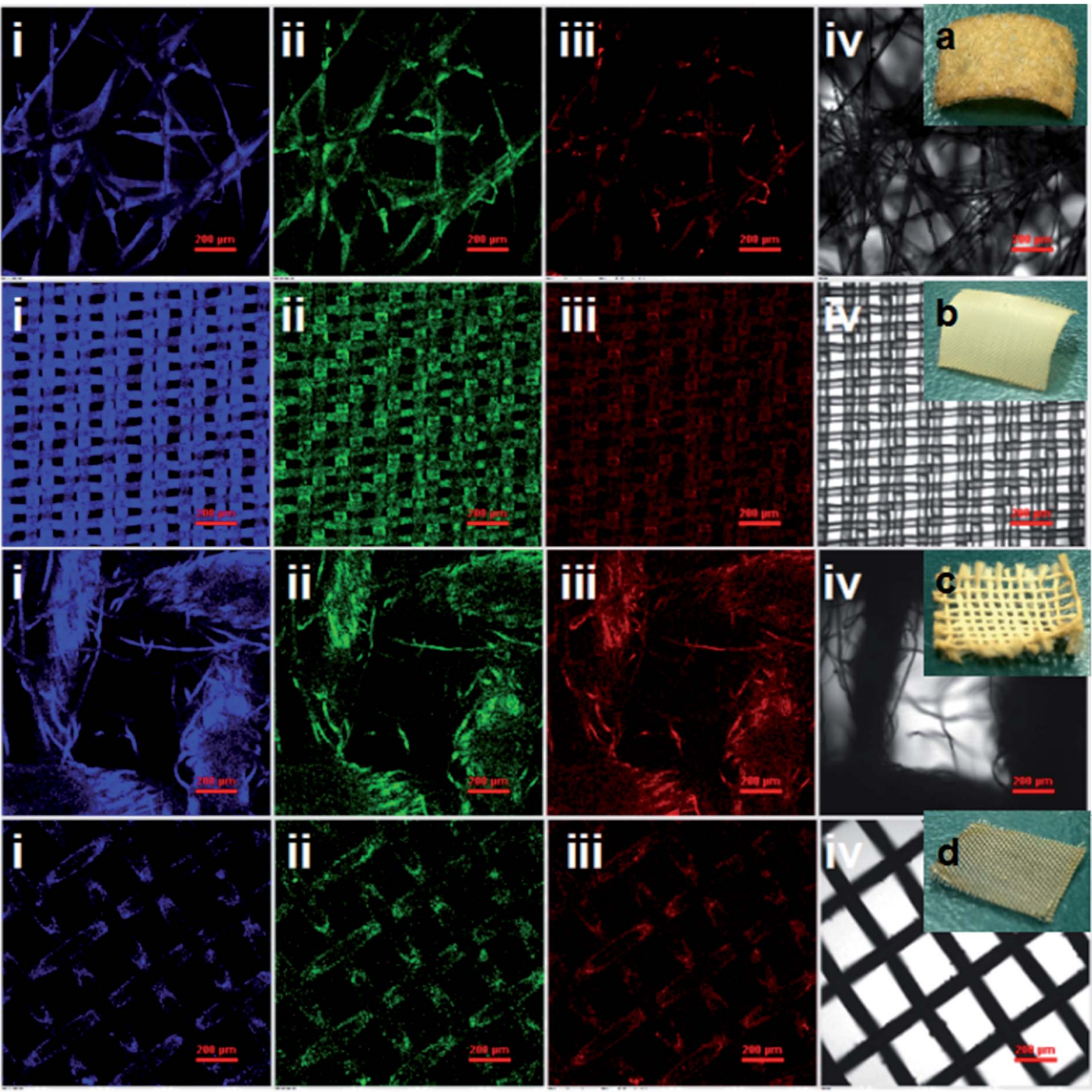

Fig. 5 Fluorescence images (i-iii) and bright-field image (iv) of textiles dyed with colloidal CDs ethanol solution. The PL textiles knitted by (a) PET, (b) nylon, (c) cotton, (d) iron fibers (insets) were captured under UV, blue, and green excitation; the fluorescent dyes used here was CDs ethanol solution. Scale bars: $200 \mu \mathrm{m}$.

such as the PET film with only $1.33 \mathrm{wt} \%$ loading fraction of CDs, which will greatly satisfy large-area applications for coating films, fabrics and solid-state flexible optoelectronic devices (Fig. 4f and Fig. S11b $\dagger$ ). ${ }^{82}$ Moreover, the good dispersion in polar solvents and excitation-wavelength-dependent PL properties of the CDs make full-color PL-tunable textiles possible. The colloidal CD ethanol solution could directly dye various textiles knitted from PET, nylon, cotton, and iron fibers, which exhibited stronger adhesion properties and brighter luminescence than conventional graphitic CDs (Fig. 5i-iv). ${ }^{83}$ Such outstanding full-color PL textiles are expected to be an ideal alternative for wearable PL devices such as smart wearable solar cells instead of costly, toxic heavy metals complexes and mutagenic, carcinogenic, persistent organic dyes. ${ }^{\mathbf{8 4}}$ Therefore, unlike traditional quantum dots, which need to be surface functionalized via quite sophisticated methods, the CDs with a large amount of hydroxyl and carbonyl groups on their surface exhibit unique optical properties, which endow them with potential large-area applications in PL coatings, smart wearable optoelectronic devices, light emitting diodes, full-color displays, and solar cells.

\section{Conclusion}

In summary, we have demonstrated a facile, green, simple and controllable approach to fabricate CDs at the oil/water interface 
using a $\mathrm{CuSO}_{4}-\mathrm{H}_{2} \mathrm{O}_{2}$ catalytic-oxidation system. This is the first time the general synthesis of CDs is proposed using an oil/water two-phase system. The characteristic feature of the synthesis is that neither harsh conditions nor surface passivation is needed. In particular, the oil-soluble small molecule styrene acts as both the reactant and oil phase medium. The yield of CDs was about $50 \mathrm{wt} \%$, which is a relatively high yield for the production of quantum dots in comparison to traditional synthetic methods. Furthermore, the self-assembly of CDs via hydrogen-bond interactions at the oil/water interface results in selfquenching-resistant and full-color tunable PL properties, which can be controlled by varying the reaction time, temperature, concentration of oxidants and assembly sizes. This will expand the novel applications of CDs in coating films, wearable optoelectronic devices, light-emitting diodes, full-color displays, and solar cells. More importantly, this synthesis for converting benzene compounds into CDs prevents the emission of greenhouse gases such as $\mathrm{CO}_{2}$ from thermal oxidation (1000 ${ }^{\circ} \mathrm{C}$ ), Fenton reactions and $\mathrm{UV} / \mathrm{O}_{3}$ oxidation systems, which will open a new area for the degradation of industrial volatile organic compound (VOC) pollutants.

\section{Conflicts of interest}

There are no conflicts to declare.

\section{Acknowledgements}

Financial support of this research from the United Innovation Program of Shanghai Commercial Aircraft Engine Fund (AR909), the Baoshan Science and Technology Project of Shanghai is appreciated.

\section{References}

1 S. Zhu, et al., Highly photoluminescent carbon dots for multicolor patterning, sensors, and bioimaging, Angew. Chem., Int. Ed., 2013, 52, 3953-3957.

2 C. Wang, D. Sun, K. Zhuo, H. Zhang and J. Wang, Simple and green synthesis of nitrogen-, sulfur-, and phosphorus-codoped carbon dots with tunable luminescence properties and sensing application, RSC Adv., 2014, 4, 54060-54065.

3 S. Zhuo, M. Shao and S. Lee, Upconversion and Down conversion Fluorescent Graphene Quantum Dots: Ultrasonic Preparation and Photocatalysis, ACS Nano, 2012, 6, 1059-1064.

4 Y. P. Sun, et al., Host-Guest Carbon Dots for Enhanced Optical Properties and Beyond, Sci. Rep., 2015, 5, 12354.

5 Y. Liu, Y. Zhao and Y. Zhang, One-step green synthesized fluorescent carbon nanodots from bamboo leaves for copper(II) ion detection, Sens. Actuators, B, 2014, 196, 647652.

$6 \mathrm{~J}$. Kwak, et al., Bright and Efficient Full-Color Colloidal Quantum Dot Light-Emitting Diodes Using an Inverted Device Structure, Nano Lett., 2012, 12, 2362-2366.

7 Q. J. Sun, et al., Bright, multicoloured light-emitting diodes based on quantum dots, Nat. Photonics, 2007, 1, 717-722.
$8 \mathrm{X}$. Bai, et al., Efficient and tuneable photoluminescent boehmite hybrid nanoplates lacking metal activator centres for single-phase white LEDs, Nat. Commun., 2014, 5, 5702.

9 G. Rukan, et al., High-Capacitance Hybrid Supercapacitor Based on Multi-Colored Fluorescent Carbon-Dots, Sci. Rep., 2017, 7, 11222.

10 Y. Liu, Y. Tian, Y. Tian, Y. Wang and W. Yang, Carbon-DotBased Nanosensors for the Detection of Intracellular Redox State, Adv. Mater., 2015, 27, 7156-7160.

$11 \mathrm{Y} . \mathrm{X} . \mathrm{Xu}$, et al., Electrophoretic analysis and purification of fluorescent single-walled carbon nanotube fragments, $J$. Am. Chem. Soc., 2004, 126, 12736-12737.

12 Y. P. Sun, et al. Quantum-Sized Carbon Dots for Bright and Colorful Photoluminescence, J. Am. Chem. Soc., 2006, 128, 7756-7757.

13 A. B. Bourlinos, et al., Photoluminescent Carbogenic Dots, Chem. Mater., 2008, 20, 4539-4541.

14 A. B. Bourlinos, et al., Surface Functionalized Carbogenic Quantum Dots, Small, 2008, 4, 455-458.

15 D. Pan, J. Zhang, Z. Li, C. Wu, X. Yan and M. Wu, Observation of $\mathrm{pH}-$, solvent-, spin-, and excitationdependent blue photoluminescence from carbon nanoparticles, Chem. Commun., 2010, 46, 3681.

16 P. C. Hsu and H. T. Chang, Synthesis of high-quality carbon nanodots from hydrophilic compounds: role of functional groups, Chem. Commun., 2012, 48, 3984-3986.

17 Y. Dong, N. Zhou, X. Lin, J. Lin, Y. Chi and G. Chen, Extraction of Electrochemiluminescent Oxidized Carbon Quantum Dots from Activated Carbon, Chem. Mater., 2010, 22, 5895-5899.

18 H. Liu, T. Ye and C. Mao, Fluorescent Carbon Nanoparticles Derived from Candle Soot, Angew. Chem., 2007, 119, 6593; Angew. Chem., Int. Ed., 2007, 46, 6473-6475.

19 Z. A. Qiao, et al., Commercially activated carbon as the source for producing multicolor photoluminescent carbon dots by chemical oxidation, Chem. Commun., 2010, 46, 8812-8814.

20 S. C. Ray, A. Saha, N. R. Jana and R. Sarkar, Fluorescent carbon nanoparticles: synthesis, characterization, and bioimaging application, J. Phys. Chem. C, 2009, 113, 1854618551.

21 L. Tian, D. Ghosh, W. Chen, S. Pradhan, X. Chang and S. Chen, Nanosized carbon particles from natural gas soot, Chem. Mater., 2009, 21, 2803-2809.

$22 \mathrm{H}$. Li, et al., One-step ultrasonic synthesis of water-soluble carbon nanoparticles with excellent photoluminescent properties, Carbon, 2011, 49, 605-609.

23 X. H. Wang, K. G. Qu, B. L. Xu, J. S. Ren and X. G. Qu, Microwave assisted one-step green synthesis of cellpermeable multicolor photoluminescent carbon dots without surface passivation reagents, J. Mater. Chem., 2011, 21, 2445-2450.

24 H. Zhu, X. Wang, Y. Li, Z. Wang, F. Yang and X. Yang, Microwave synthesis of fluorescent carbon nanoparticles with electrochemiluminescence properties, Chem. Commun., 2009, 5118-5120. 
25 B. Zhang, C. Y. Liu and Y. Liu, A Novel One-Step Approach to Synthesize Fluorescent Carbon Nanoparticles, Eur. J. Inorg. Chem., 2010, 4411-4414.

26 Y. M. Long, et al., Shifting and non-shifting fluorescence emitted by carbon nanodots, J. Mater. Chem., 2012, 22, 5917-5920.

27 L. Bao, et al., Electrochemical Tuning of Luminescent Carbon Nanodots: From Preparation to Luminescence Mechanism, Adv. Mater., 2011, 23, 5801-5806.

28 B. Zhang, C. Y. Liu and Y. Liu, A Novel One-Step Approach to Synthesize Fluorescent Carbon Nanoparticles, Eur. J. Inorg. Chem., 2010, 4411-4414.

29 H. Nie, M. Li, Q. Li, S. Liang, Y. Tan, L. Sheng, et al., Carbon dots with continuously tunable full-color emission and their application in ratiometric pH sensing, Chem. Mater., 2014, 26, 3104-3112.

30 L. Tang, R. Ji, X. Li, G. Bai, C. P. Liu, J. Hao, et al., Deep ultraviolet to near-Infrared emission and photoresponse in layered N-doped graphene quantum dots, ACS Nano, 2014, 8, 6312-6320.

31 S. Zhu, J. Zhang, S. Tang, C. Qiao, L. Wang, H. Wang, et al., Surface chemistry routes to modulate the photoluminescence of graphene quantum dots: from fluorescence mechanism to up-conversion bioimaging applications, Adv. Funct. Mater., 2012, 22, 4732-4740.

32 S. Zhu, Q. Meng, L. Wang, J. Zhang, Y. Song, H. Jin, et al., Highly photoluminescent carbon dots for multicolor patterning, sensors, and bioimaging, Angew. Chem., Int. Ed., 2013, 52, 3953-3957; Angew. Chem., 2013, 125, 40454049.

33 H. Tetsuka, R. Asahi, A. Nagoya, K. Okamoto, I. Tajima, R. Ohta, et al., Optically tunable amino-functionalized graphene quantum dots, Adv. Mater., 2012, 24, 5333.

34 S. K. Bhunia, A. Saha, A. R. Maity, S. C. Ray and N. R. Jana, Carbon nanoparticle-based fluorescent bioimaging probes, Sci. Rep., 2013, 3, 1473.

35 Y. Lu, L. Zhang and H. Lin, The use of a microreactor for rapid screening of the reaction conditions and investigation of the photoluminescence mechanism of carbon dots, Chem.-Eur. J., 2014, 20, 4246.

36 K. Jiang, S. Sun, L. Zhang, Y. Lu, A. Wu, C. Cai, et al., Red, green, and blue luminescence by carbon dots: full-color emission tuning and multicolor cellular imaging, Angew. Chem., 2015, 127, 5450-5453.

37 C. L. Chiang, et al., Red-Emitting Fluorenes as Efficient Emitting Hosts for Non-Doped, Organic Red-LightEmitting Diodes, Adv. Funct. Mater., 2005, 15, 231-238.

38 M. Shimizu, Y. Takeda, M. Higashi and T. Hiyama, 1,4Bis(alkenyl)-2,5-dipiperidinobenzenes: Minimal Fluorophores Exhibiting Highly Efficient Emission in the Solid State, Angew. Chem., Int. Ed., 2009, 48, 3653-3656.

39 M. Shimizu, et al., Dibenzosiloles and 12H-Indololo[3,2-d] naphtho[1,2-b][1]siloles: Exploration of Organic Chromophores Exhibiting Efficient Solid-State Fluorescence, Chem. Rec., 2015, 15, 73.

$40 \mathrm{X}$. Wang, et al., A general strategy for nanocrystal synthesis, Nature, 2005, 437, 121-124.
41 Z. Nie, A. Petukhova and E. Kumacheva, Properties and emerging applications of self-assembled structures made From inorganic nanoparticles, Nat. Nanotechnol., 2010, 5, 15-25.

42 M. Brust, D. Bethell, C. J. Kiely and D. J. Schiffrin, Selfassembled gold nanoparticle thin films with nonmetallic optical and electronic properties, Langmuir, 1998, 14, 5425-5429.

43 M. Segev-Bar, A. Landman, M. Nir-Shapira, G. Shuster and H. Haick, Tunable touch sensor and combined sensing platform: toward nanoparticle-based electronic skin, ACS Appl. Mater. Interfaces, 2013, 5, 5531-5541.

$44 \mathrm{~S}$. L. Hu, et al., One-step synthesis of fluorescent carbon nanoparticles by laser irradiation, J. Mater. Chem., 2009, 19, 484-488.

45 D. Tan, et al., Simple synthesis of ultra-small nanodiamonds with tunable size and photoluminescence, Carbon, 2013, 62, 374-381.

46 A. Hill Stephen, D. Benito-Alifonso, J. Morgan David, A. Davis Sean, M. Berrya and M. Carmen Galan, Nanoscale, 2016, 8, 18630.

47 Y. Yang, D. Wu, S. Han, P. Hu and R. Liu, Bottom-up fabrication of photoluminescent carbon dots with uniform morphology via a soft-hard template approach, Chem. Commun., 2013, 49, 4920-4922.

48 B. De and N. Karak, A green and facile approach for the synthesis of water soluble fluorescent carbon dots from banana juice, $R S C$ Adv., 2013, 3, 8286-8290.

49 Q. Liang, W. Ma, Y. Shi, Z. Li and X. Yang, Easy synthesis of highly fluorescent carbon quantum dots from gelatin and their luminescent properties and applications, Carbon, 2013, 60, 421-428.

50 A. M. Panich, et al., Structure and bonding in fluorinated nanodiamond, J. Phys. Chem. C, 2010, 114, 774-782.

51 S. Osswald, G. Yushin, V. Mochalin, S. O. Kucheyev and Y. Gogotsi, Control of $\mathrm{sp}^{2} / \mathrm{sp}^{3}$ carbon ratio and surface chemistry of nanodiamond powders by selective oxidation in air, J. Am. Chem. Soc., 2006, 128, 11635-11642.

52 O. Shenderova, et al., Surface chemistry and properties of ozone-purified detonation nanodiamonds, J. Phys. Chem. C, 2011, 115, 9827-9837.

53 K. J. D. MacKenzie and M. E. Smith. Multinuclear solid-state NMR of inorganic materials, Pergamon, Oxford, 1st edn, 2002.

54 G. Cunningham, et al., Diamond Relat. Mater., 2008, 17, 650654.

55 M. Panich Alexander, et al., J. Phys. Chem. C, 2010, 114, 774782.

$56 \mathrm{X}$. Li, et al., Intercrossed Carbon Nanorings with Pure Surface States as Low-Cost and Environment-Friendly Phosphors for White-Light-Emitting Diodes, Angew. Chem., Int. Ed., 2015, 54, 1759-1764.

57 J. C. G. Esteves da Silva and H. M. R. Goncalves, Analytical and bioanalytical applications of carbon dots, Trends Anal. Chem., 2011, 30, 1327-1336.

58 Y. Lin, H. Skaff, T. Emrick, A. D. Dinsmore and T. P. Russell, Nanoparticle Assembly and Transport at Liquid-Liquid Interfaces, Science, 2003, 299, 226-229. 
59 H. Lin Sheng, M. Lin Chi and G. L. Horng, Operating characteristics and kinetic studies of surfactant wastewater treatment by fenton oxidation, Water Research, 1999, 33, 1735-1741.

60 Y. Luo, O. Miklds, K. Kenneth and R. Irving, Epstein Mechanistic Study of Oscillations and Bistability in the $\mathrm{Cu}$ (II)-Catalyzed Reaction between $\mathrm{H}_{2} \mathrm{O}_{2}$ and KSCN, J. Am. Chem. Soc., 1989, 111, 4548-4552.

$61 \mathrm{H}$. Lee, H. Lee and C. Lee, Degradation of diclofenac and carbamazepine by the copper(II)-catalyzed dark and photoassisted Fenton-like systems, Chem. Eng. J., 2014, 245, 258264.

62 A. Kossiakoff and F. O. Rice, Thermal Decomposition of Hydrocarbons, Resonance Stabilization and Isomerization of Free Radicals, J Am Chem Soc, 1943, 65, 590-595.

63 Z. Su, W. Zhou and Y. Zhang, New insight into the soot nanoparticles in a candle flame, Chem. Commun., 2011, 47, 4700-4702.

64 P. Badziag, W. S. Verwoerd, W. P. Ellis and N. R. Greiner, Nanometre-sized diamonds are more stable than Graphite, Nature, 1990, 343, 244-245.

65 I. I. Vlasov, A. A. Shiryaev, T. Rendler, et al., Molecular-sized fluorescent nanodiamonds, Nat. Nanotechnol., 2014, 9, 5458.

66 Y. Li, et al., A Reduction-Pyrolysis-Catalysis Synthesis of Diamond, Science, 1998, 281, 246-247.

67 H. Li, et al., Water-Soluble Fluorescent Carbon Quantum Dots and Photocatalyst Design, Angew. Chem., Int. Ed., 2010, 49, 4430-4434.

68 S. K. Cushing, M. Li, F. Huang and N. Wu, Origin of Strong Excitation Wavelength Dependent Fluorescence of Graphene Oxide, ACS Nano, 2014, 8, 1002-1013.

69 H. Li, et al., Water-Soluble Fluorescent Carbon Quantum Dots and Photocatalyst Design, Angew. Chem., Int. Ed., 2010, 49, 4430-4434.

70 L. Bao, et al., Photoluminescence-Tunable Carbon Nanodots: Surface-State Energy-Gap Tuning, Adv. Mater., 2015, 27, 1663-1667.

$71 \mathrm{~S}$. Kim, et al., Size-dependent radiative decay processes in graphene quantum dots, Appl. Phys. Lett., 2012, 101, 163103.

$72 \mathrm{~J}$. Zhou, et al., An Electrochemical Avenue to Blue Luminescent Nanocrystals from Multiwalled Carbon Nanotubes (MWCNTs), J. Am. Chem. Soc., 2007, 129, 744745.
73 L. Tang, et al., Deep ultraviolet photoluminescence of watersoluble self-passivated graphene quantum dots, ACS Nano, 2012, 6, 5102-5110.

74 Q. L. Zhang, et al., Reactivity of Large Carbon Clusters: Spheroidal Carbon Shells and Their Possible Relevance to the Formation and Morphology of Soot, J. Phys. Chem., 1986, 90, 525-528.

$75 \mathrm{~K}$. Jiang, et al., Red, green, and blue luminescence by carbon dots: full-color emission tuning and multicolor cellular imaging, Angew. Chem., Int. Ed., 2015, 54, 5360-5363.

76 X. Wang, K. Qu, B. Xu, J. Ren and X. Qu, Multicolor luminescent carbon nanoparticles: Synthesis, supramolecular assembly with porphyrin, intrinsic peroxidase-like catalytic activity and applications, Nano Res., 2011, 4, 908-920.

77 K. Jiang, S. Sun, L. Zhang, et al., Red, green, and blue luminescence by carbon dots: full-color emission tuning and multicolor cellular imaging, Angew. Chem., Int. Ed., 2015, 54, 5360-5363.

78 M. Wang, R. Sun, Q. Wang, et al., Effects of C-Related Dangling Bonds and Functional Groups on the Fluorescent and Electrochemiluminescent Properties of Carbon-Based Dots, Chem.-Eur. J., 2018, 24, 4250-4254.

79 M. J. Krysmann, et al., Formation Mechanism of Carbogenic Nanoparticles with Dual Photoluminescence Emission, J. Am. Chem. Soc., 2012, 134, 747-750.

80 Z. Xie, F. Wang and C. Liu, Organic-Inorganic Hybrid Functional Carbon Dot Gel Glasses, Adv. Mater., 2012, 24, 1716-1721.

81 Y. Shishino, T. Yonezawa, S. Udagawa, K. Hase and H. Nishihara, Preparation of Optical Resins Containing Dispersed Gold Nanoparticles by the Matrix Sputtering Method, Angew. Chem., Int. Ed., 2011, 50, 703-705.

$82 \mathrm{R}$. Kabe and C. Adachi, Organic long persistent luminescence, Nature, 2017, 550, 384-387.

83 S. Qu, X. Wang, Q. Lu, et al., A Biocompatible Fluorescent Ink Based on Water-Soluble Luminescent Carbon Nanodots, Angew. Chem., 2012, 124, 12381-12384.

84 H. Jinno, K. Fukuda, T. Someya, et al., Stretchable and waterproof elastomer-coated organic photovoltaics for washable electronic textile applications, Nat. Energy, 2017, 2, 780-785. 